\title{
Chest Compression Metrics During Manual Cardiopulmonary Resuscitation: a Manikin Study
}

\author{
Sofía Ruiz de Gauna ${ }^{1}$, Digna M González-Otero ${ }^{1}$, James K Russell ${ }^{2}$, Jesus Ruiz ${ }^{1}$, Sara Pelayo ${ }^{1}$, \\ Purificación Saiz ${ }^{1}$ \\ ${ }^{1}$ University of the Basque Country (UPV/EHU), Bilbao, Spain \\ ${ }^{2}$ Oregon Health \& Science University (OHSU), Portland OR, USA
}

\begin{abstract}
Chest compression quality during cardiopulmonary resuscitation $(C P R)$ is defined by adequate rate and depth, with complete chest recoil. Other metrics are duty cycle, or the recently introduced release velocity. However, the relationship between metrics is not sufficiently understood.

Our aim was to design and validate tools for the automated annotation and analysis of compression metrics during manual CPR in a simulated manikin setting.

Eleven volunteers delivered chest compressions on a manikin equipped with a distance sensor to measure chest displacement. Compression depth signal was acquired during 2-min sessions, with different chest stiffness and target compression rates. The annotated metrics for each compression were: compression and decompression duration $\left(T_{c}, T_{d}\right)$, compression depth $\left(d_{p}\right)$, duty cycle $(D C)$, compression velocity $(\mathrm{CV})$, and release velocity $(R V)$.

We annotated 31451 compressions in 132 recordings, and analyzed the distributions of the annotated metrics: $d_{p}$ decreased with increasing rate and stiffness. $D C, C V$ and $R V$ increased with rate, and differed with stiffness. $C V$ and $R V$ showed a strong linear correlation with the ratio $d_{p} / T_{c}$ and $d_{p} / T_{d}$, respectively.

The study provided a reliable framework for the characterization of chest compressions during manual $C P R$, and could be extended to human data.
\end{abstract}

\section{Introduction}

Quality of chest compressions during cardiopulmonary resuscitation (CPR) plays a critical role in the treatment of out-of-hospital $(\mathrm{OOH})$ cardiac arrest. Delivering early and high-quality chest compressions to the patient generates a minimal but critical amount of blood flow to the heart and the brain, and contributes to enhance survival.

Current recommendations for adult high-quality chest compressions are: depth of at least $50 \mathrm{~mm}$, without exceeding $60 \mathrm{~mm}$; rate between 100 and 120 compressions per minute (cpm); allowing complete chest recoil between compressions, and minimizing interruptions [1]. In addition, a duty cycle of $50 \%$ is recommended, that is, half of the compression cycle should be spent compressing the chest. Target values for compression quality metrics have been determined by clinical findings from animal and human studies, and have evolved through the years in parallel to clinical evidence [2,3].

However, resuscitation committees state the need of additional studies on the relationship between compression parameters (especially depth and rate), and how optimal values vary in relation to different patient stiffness. In addition, release velocity (RV) has been recently proposed as a novel quality metric [4], although its relation with current quality metrics is not sufficiently understood.

The aim of this study was to design tools for automated annotation and analysis of chest compression metrics and to test them in a simulated and controlled manikin setting. We also wanted to study the relationship between the current and newly proposed chest compression metrics.

\section{Materials and methods}

\subsection{Experimental setup}

A resuscitation manikin (Resusci-Anne QCPR, Laerdal Medical, Norway) was equipped with a resistive distance sensor (SP1-4, Celesco, USA) for continuous recording of the chest displacement. The manikin included three different springs to model human chest stiffness: soft, standard, and hard, accounting for the significant variability of chest stiffness between patients suffering from cardiac arrest [5].

Compression depth signal obtained from the distance sensor was digitized using a data acquisition card (USB NI-6211, National Instruments, USA) connected to a laptop computer. Signals were stored in Matlab ${ }^{\circledR}$ (Mathworks, USA) format, with a sampling frequency of $500 \mathrm{~Hz}$ and a 16-bit resolution. Figure 1 shows the experimental setup used in the study: the acquisition card connected to the laptop, and the manikin torso with the three springs: from left to right, yellow (soft stiffness), steel grey (standard), and blue (hard). 


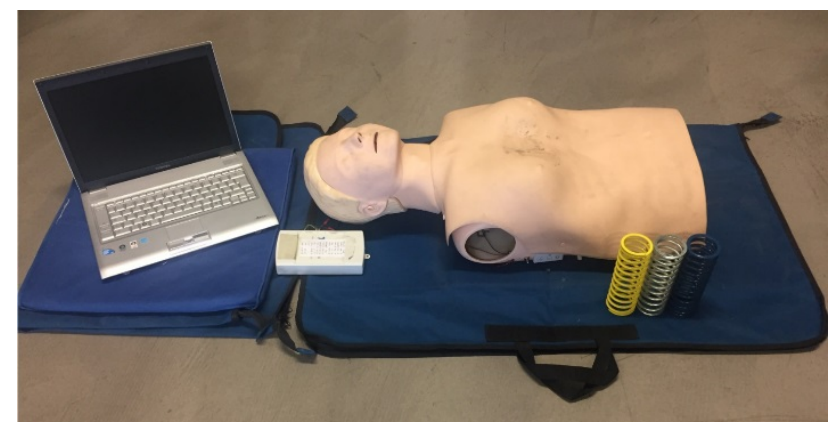

Figure 1. Experimental setup. From left to right: laptop computer, acquisition card, manikin torso, and springs for simulating different chest stiffness.

Eleven volunteers received a basic CPR course and signed an informed consent form before initiating the experimental sessions. They were instructed to deliver continuous chest compressions to the manikin during 2 minutes without pauses. The degree of stiffness and the target compression rate were randomly selected for each experiment. Target compression rate was $80,100,120$, or $140 \mathrm{cpm}$, and was guided with a metronome. Volunteers were coached to avoid leaning, i.e., to allow complete recoil of the chest between chest compressions.

\subsection{Data annotation}

We developed Matlab ${ }^{\circledR}$ tools for the automated annotation of different metrics associated to each chest compression instance in the recorded compression depth signal. These tools also allowed visual inspection of the records and of the annotated metrics. Figure 2 shows an example of the resulting annotations for a single chest compression.

First, we obtained the continuous chest velocity signal (Figure 2, top) as the first derivative of the compression depth signal (bottom). We used these two signals to identify some fiducial points and metrics for each compression.

Second, we applied a simple peak detector to the compression depth signal to identify compressions with a depth of at least $10 \mathrm{~mm}$.

For each compression instance, the following fiducial points were annotated (Figure 2, bottom panel):

- Instant of the maximum negative depletion of the compression depth signal, $t_{\mathrm{dp}}$. This instant corresponded to a negative to positive zero crossing instant in the chest velocity.

- $\quad$ Start and end of the compression cycle, $t_{\mathrm{s}}$ and $t_{\mathrm{e}}$, respectively, which corresponded to null chest velocity.

- Effective start of the compression cycle, $t_{\mathrm{s}_{-} \text {ef }}$, defined as the point where a certain depth, $d_{\mathrm{th}}$, is achieved.
- Effective end of the compression cycle, $t_{\text {e_ef }}$, defined as the point where the manikin chest was released up to $d_{\mathrm{th}}$.

The annotated metrics were:

- Compression depth, $d_{\mathrm{p}}$ : value of the compression depth signal at the time $t_{\mathrm{dp}}$.

- Compression time, $T_{\mathrm{c}}$ : time required to achieve $d_{\mathrm{p}}$, from the effective start of the compression:

$$
T_{\mathrm{c}}=t_{\mathrm{dp}}-t_{\mathrm{s} \text { ef }}
$$

- Decompression time, $T_{\mathrm{d}}$ : time required to release the chest:

$$
T_{d}=t_{e_{-} e f}-t_{d p}
$$

- Compression velocity, $C V$ : maximum chest velocity during the compression time.

- Release velocity, $R V$ : maximum chest velocity during the decompression time.

- Duty cycle, $D C$ : percentage of time spent in compression relative to the duration of the compression cycle:

$$
D C=100 \cdot T_{c} /\left(T_{c}+T_{d}\right)
$$
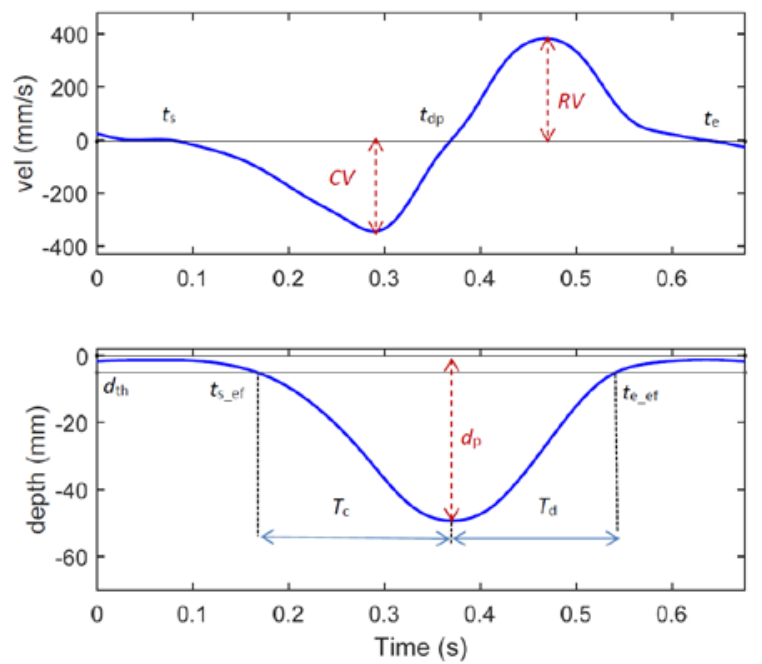

Figure 2. Example of annotated fiducial points and metrics for one chest compression cycle.

\subsection{Data analysis}

As the annotated metrics did not pass the Lilliefors normality test, their distributions were characterized by their median and $25^{\text {th }}$ and $75^{\text {th }}$ percentiles $\left(P_{25}-P_{75}\right)$. Distribution of computed metrics was analyzed in relation to stiffness and compression rate. We applied KruskalWallis test for comparison between groups, and a pvalue $<0.05$ was considered significant.

We also assessed the relationship between compression depth, release velocity and decompression time using univariate linear regression and Pearson's correlation 
coefficient, $r$. Our hypothesis was that there would be a strong linear relationship between $R V$ and the ratio $d_{p} / T_{d}$, A similar analysis was conducted with compression velocity, $C V$ and the ratio $d_{p} / T_{c}$.

We also classified chest compressions individually as high-quality or low-quality chest compressions to analyze the power of $R V$ as a reliable quality parameter. For this analysis, we classified as high-quality chest compressions those with depth between 50 and $60 \mathrm{~mm}$ and compression rate between 100 and $120 \mathrm{cpm}$.

\section{Results}

We visually inspected all the episodes after the automated annotation. We discarded 315 compressions that did not exceeded the $10 \mathrm{~mm}$ threshold. A total of 31451 chest compressions were annotated in 132 episodes, with a mean 238 (47) compressions per episode.

The threshold $d_{\text {th }}$ for determining the effective start and end of the compression cycle was fixed at $5 \mathrm{~mm}$.

Figure 3 shows the distributions of some of the analyzed metrics. For the whole population, median $\left(P_{25}-P_{75}\right) d_{p}$ was 48.7 (43.1-53.7) mm; $D C$ was 50.9 (48.7-52.8) \%; $R V$ was 485.0 (423.4-541.4) mm/s and $C V$ was 470.0 (406.5$542.5) \mathrm{mm} / \mathrm{s}$.
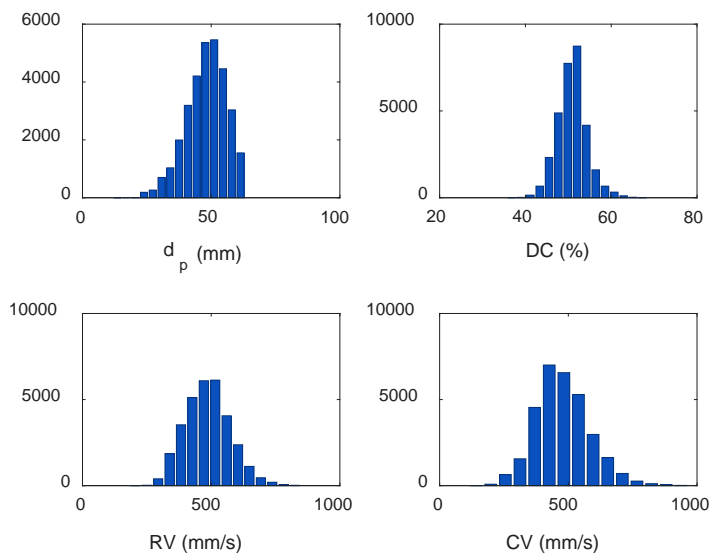

Figure 3. Histograms of some of the computed compression metrics.

\subsection{Compression metrics in relation to stiffness and compression rate}

Table 1 shows the variation of the computed compression metrics with increasing stiffness. Results were statistically different for all the metrics.

Figure 4 shows the variation of metrics as a function of compression rate. Continuous line represents the median value, while dashed lines represents $P_{25}$ and $P_{75}$. There were significant differences depending on target compression rate for all the metrics.
Table 1. Compression metrics for different grades of stiffness. $d_{p}$ in mm; $D C$ in \%; $R V$ and $C V$ in $\mathrm{mm} / \mathrm{s}$. Values are expressed as integers for clarity

\begin{tabular}{|c|c|c|c|}
\hline \multirow{2}{*}{ Metric $^{1}$} & \multicolumn{3}{|c|}{ Stiffness } \\
\hline & Soft & Standard & Hard \\
\hline$d_{p}$ & 54 (48-57) & 49 (44-53) & 45 (40-49) \\
\hline$D C$ & $51 \quad(49-52)$ & 52 (50-53) & 50 (48-53) \\
\hline$R V$ & $502(455-550)$ & $466(406-527)$ & $478(414-549)$ \\
\hline$C V$ & $448(417-540)$ & 447 (392-524) & 489 (413-578) \\
\hline
\end{tabular}
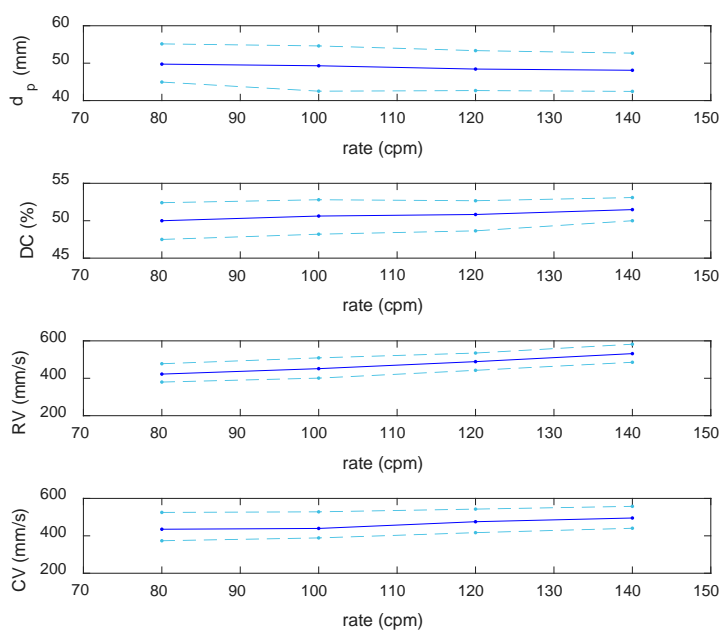

Figure 4. Variation of compression metrics as a function of compression rate.

\subsection{Relationship between compression depth and chest velocity}

Release velocity $R V$ showed a high linear relationship with the ratio $d_{p} / T_{d}$ when all chest compressions were considered. The correlation coefficient was 0.97 . Compression velocity $C V$ showed also a high linear relationship with the ratio $d_{p} / T_{c}$, with a correlation coefficient of 0.94 .

Figure 5 shows the scatter plot and the models fitted to all values jointly. According to this, a good approximation to the actual release velocity as a function of the ratio depth-decompression time would be:

$$
R V=1.32 \cdot \frac{d_{p}}{T_{d}}
$$

A similar approximation could explain the relation between $C V$ and the ratio $d_{p} / T_{c}$ :

$$
C V=1.33 \cdot \frac{d_{p}}{T_{c}}
$$



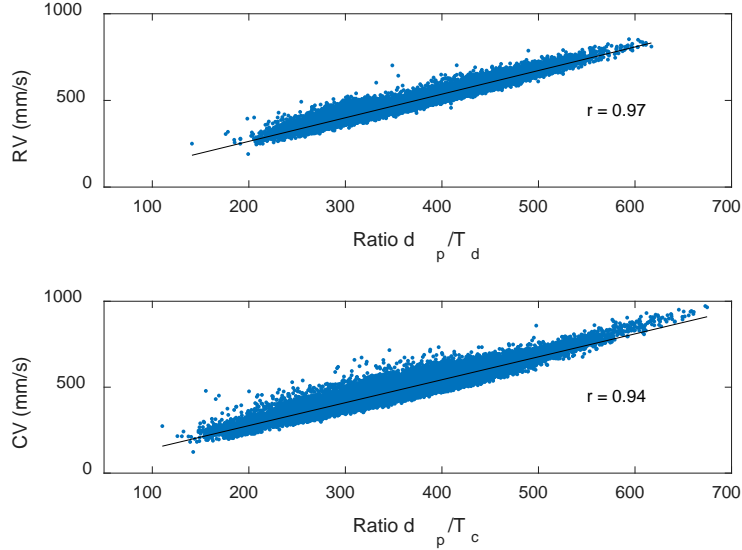

Figure 5. Linear relationship between $R V$ and $d_{p} / T_{d}$ (top) and between $C V$ and $d_{p} / T_{c}$ (bottom).

Finally, Figure 6 shows the distribution of RV for highquality and low quality chest compressions.

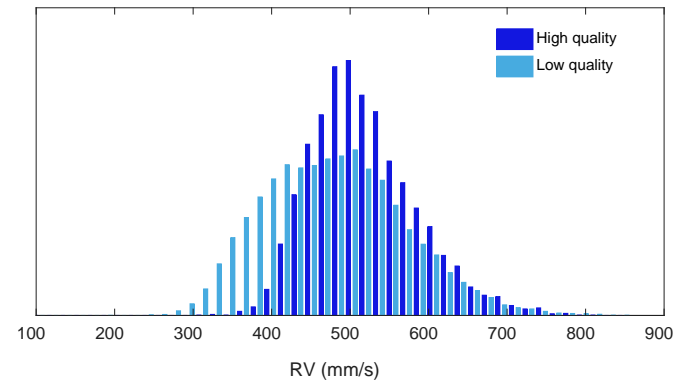

Figure 6. Histograms of $R V$ for high-quality (dark) and low-quality (light) chest compressions.

\section{Discussion and conclusions}

Extensive analysis of chest compression parameters could contribute to a better understanding of CPR dynamics and the relationship between current and novel quality metrics. Our study provided automated tools for this characterization that could be useful for the analysis of human CPR data.

Our results confirmed that the minimum recommended $50 \mathrm{~mm}$ compression depth is difficult to achieve, at least in a manikin model, and that depth decreases with rate and with stiffness. Chest velocities during compression and decompression also depended on stiffness and rate. We also found a linear correlation between velocity and depth, but results did not confirm the potential value of RV as a reliable metric to differentiate between good and poor quality chest compressions. In any case, it should be considered an additional compression metric, complementary to rate and depth.

Availability of compression depth signal in human OHCA data requires the use of commercial CPR feedback devices, mostly based on accelerometers [6]. Deriving depth from acceleration is not straightforward and requires measuring chest force to account for chest release [6]. Nevertheless, the experimental setup presented here allows the reliable assessment of the relationship among different compression metrics, avoiding the uncertainty associated to the estimate of compression depth signal.

\section{Conclusions}

This study provided a reliable framework for the characterization of chest compressions during manual CPR. Assessing the relationship among chest compression metrics could lead to a better understanding of the optimal chest compression procedure.

\section{Acknowledgements}

This work was supported by the Basque Government (Basque Country, Spain) through the grant IT1087-16. The authors thank all volunteers participating in collecting the data for the study.

\section{References}

[1] Monsieurs KG, Nolan JP, Bossaert LL, et al. European Resuscitation Council guidelines for resuscitation 2015. Section 1. Executive summary. Resuscitation 2015;95:1-80.

[2] Meaney PA, Bobrow BJ, Mancini ME, et al. Cardiopulmonary resuscitation quality: improving cardiac resuscitation outcomes both inside and outside the hospital: a consensus statement from the American Heart Association. Circulation 2013;128:417-35.

[3] Perkins GV, Travers AH, Berg RA, et al. Part 3: Adult basic life support and automated external defibrillation 2015. International consensus on cardiopulmonary resuscitation and emergency cardiovascular care science with treatment recommendations. Resuscitation 2015;95:e43-e69.

[4] Cheskes S, Common MR, Byers AP, Zhan C, Silver A, Morrison LJ. The association between chest compression release velocity and outcomes from out-of-hospital cardiac arrest. Resuscitation 2015;86:38-43.

[5] Tomlinson AE, Nysaether J, Kramer-Johansen J, Steen P, and Dorph E. Compression force-depth relationship during out-of-hospital cardiopulmonary resuscitation. Resuscitation 2007;72(3); 364-370.

[6] Ruiz de Gauna S, González-Otero DM, Ruiz J, Russell JK. Feedback on the rate and depth of chest compressions during cardiopulmonary resuscitation using only accelerometers. PLOS ONE 2016;11:e0150139.

Address for correspondence.

Sofía Ruiz de Gauna

Department of Communications Engineering

School of Engineering

Alameda Urquijo, s/n

48013-Bilbao (Spain)

sofia.ruizdegauna@ehu.eus 